John Carroll University

Carroll Collected

2018 Faculty Bibliography

Faculty Bibliographies Community Homepage

2018

\title{
Quantitative analysis of a brass alloy using CF- LIBS and a laser ablation time-of-flight mass spectrometer
}

\author{
Nasar Ahmed \\ Quaid-i-Azam University Islamabad, Pakistan \\ M. Abdullah \\ Quaid-i-Azam University Islamabad, Pakistan \\ Rizwan Ahmed \\ Quaid-i-Azam University Islamabad, Pakistan \\ Naveed K. Piracha \\ John Carroll University, npiracha@jcu.edu \\ M. Aslam Baig \\ Quaid-i-Azam University Islamabad, Pakistan
}

Follow this and additional works at: https://collected.jcu.edu/fac_bib_2018

Part of the Physics Commons

\section{Recommended Citation}

Ahmed, Nasar; Abdullah, M.; Ahmed, Rizwan; Piracha, Naveed K.; and Baig, M. Aslam, "Quantitative analysis of a brass alloy using CF- LIBS and a laser ablation time-of-flight mass spectrometer" (2018). 2018 Faculty Bibliography. 2.

https://collected.jcu.edu/fac_bib_2018/2

This Article is brought to you for free and open access by the Faculty Bibliographies Community Homepage at Carroll Collected. It has been accepted for inclusion in 2018 Faculty Bibliography by an authorized administrator of Carroll Collected. For more information, please contact connell@jcu.edu. 


\title{
Quantitative analysis of a brass alloy using CF-LIBS and a laser ablation time-of-flight mass spectrometer
}

\author{
Nasar Ahmed ${ }^{1,2}$, M Abdullah $^{1}$, Rizwan Ahmed ${ }^{1}$, N K Piracha $^{3}$ \\ and M Aslam Baig ${ }^{1,2}$ \\ ${ }^{1}$ National Centre for Physics, Quaid-i-Azam University Campus, 45320 Islamabad, Pakistan \\ ${ }^{2}$ Department of Physics, University of Azad Jammu and Kashmir, Muzaffarabad 13100, Azad Kashmir \\ 3 Department of Physics, John Carroll University, University Heights, OH-44118, United States of America
}

\begin{abstract}
We present a quantitative analysis of a brass alloy using laser induced breakdown spectroscopy, energy dispersive $\mathrm{x}$-ray spectroscopy (EDX) and laser ablation time-of-flight mass spectrometry (LA-TOF-MS). The emission lines of copper ( $\mathrm{Cu} \mathrm{I}$ ) and zinc ( $\mathrm{Zn} \mathrm{I})$, and the constituent elements of the brass alloy were used to calculate the plasma parameters. The plasma temperature was calculated from the Boltzmann plot as $(10000 \pm 1000) \mathrm{K}$ and the electron number density was determined as $(2.0 \pm 0.5) \times 10^{17} \mathrm{~cm}^{-3}$ from the Stark-broadened $\mathrm{Cu}$ I line as well as using the Saha-Boltzmann equation. The elemental composition was deduced using these techniques: the Boltzmann plot method ( $70 \% \mathrm{Cu}$ and $30 \% \mathrm{Zn}$ ), internal reference self-absorption correction $(63.36 \% \mathrm{Cu}$ and $36.64 \% \mathrm{Zn}), \mathrm{EDX}(61.75 \% \mathrm{Cu}$ and $38.25 \% \mathrm{Zn}$ ), and LA-TOF (62\% $\mathrm{Cu}$ and $38 \% \mathrm{Zn}$ ), whereas, the certified composition is (62\% $\mathrm{Cu}$ and $38 \% \mathrm{Zn}$ ). It was observed that the internal reference self-absorption correction method yields analytical results comparable to that of EDX and LA-TOF-MS.
\end{abstract}

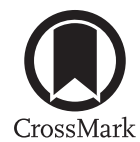

Keywords: CF-LIBS, internal reference self-absorption correction (IRSAC), brass, quantitative analysis, plasma parameters, energy dispersive x-ray spectroscopy (EDX), laser ablation time-of-flight mass spectrometry (LA-TOF-MS)

(Some figures may appear in colour only in the online journal)

\section{Introduction}

Laser induced breakdown spectroscopy (LIBS) is an analytical technique that is extensively used for the qualitative and quantitative analysis of different materials and samples of specific interests [1-5]. In this technique, a high power laser beam is focused on the surface of any material that ablates a fraction of the material and produces a plasma plume that contains neutral atoms, ions and electrons. The emission spectrum of the laser-produced plasma plume yields important information about the constituent elements and plasma parameters. However, the emission depends on the wavelength of the exciting laser light, the distance of the target sample from the laser, pulse duration, composition of the sample and ambient conditions. The LIBS technique can be used to analyse any material existing in any phase such as liquid, solid or gas [611]. It is a fast and a non-destructive method for analysing even a much minute quantity of precious samples and there is no need for any specific sample preparation. Due to these advantages, the LIBS analytical technique has been applied in several fields, in particular medical, agriculture, material processing and in environmental pollution monitoring [12-15].

Ciucci et al [16] demonstrated the LIBS self-calibration technique for quantitative analysis, whereas the LIBS calibration free method (CF-LIBS) was developed by Gomba et al [17] and has been extensively used for the compositional analysis of numerous samples [18-20]. The internal reference-external standard iteration correction method was 


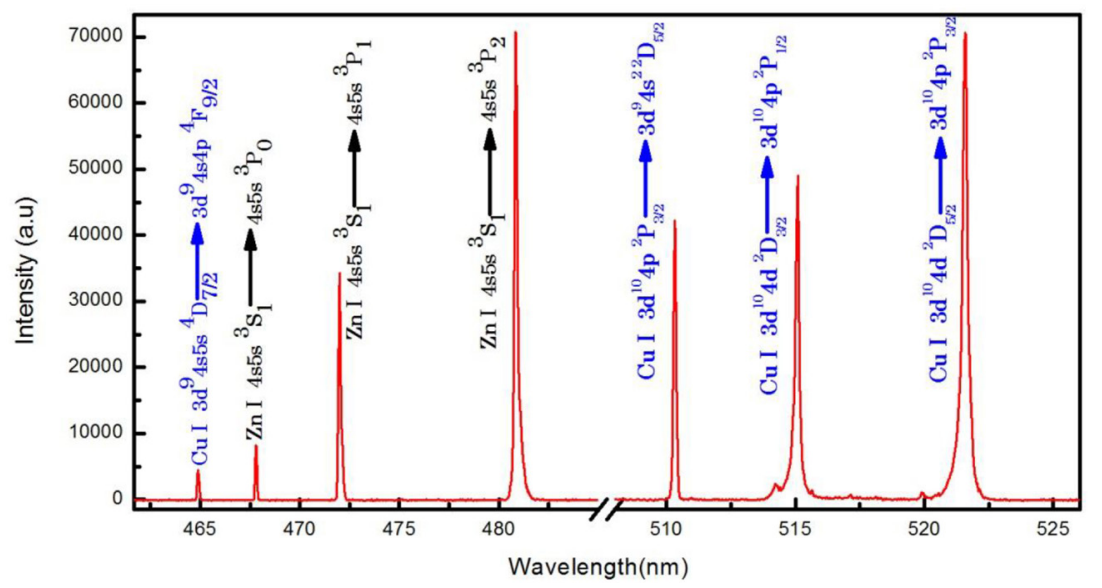

Figure 1. Optical emission spectrum of the laser-produced brass plasma, covering the spectral region $463-527 \mathrm{~nm}$.

proposed by Dong et al [21], in which self-absorption effects can be corrected by selecting an internal reference line to correct the intensities of the other spectral lines. A computer programme, a genetic algorithm, has also been used to estimate the plasma temperature. A slightly different technique, known as internal reference self-absorption correction (IRSAC) was introduced by Sun et al [22, 23], which is based on an iteration procedure to correct the emission line intensities with reference to an internal standard line. An algorithm based on the curve of growth (COG) method has also been proposed for self-absorption corrections of the spectral lines. Although the quantitative analysis by the COG method gives improved results, it requires much extensive computational work.

Although LIBS based studies on brass alloys having different compositions have been reported in the literature [24-28], the main objectives of the present work are to exploit the LIBS techniques for the quantitative analysis of a brass alloy containing copper and zinc of known compositions, $62 \%$ and $38 \%$, respectively, and to compare the extracted results with the certified compositions, as well as with that determined using well established analytical techniques such as x-ray spectroscopy (EDX) and laser ablation time-of-flight mass spectrometry (LA-TOF-MS). It is observed that the IRSAC results are in good agreement with the certified compositions and that of the EDX and LA-TOF-MS results.

\section{Experimental details}

The experimental details for recording the optical emission spectrum of the laser-produced plasma are the same as described in our earlier papers [29-32]. In brief, the laser-produced brass alloy plasma was generated using a high power $Q$-switched Nd:YAG laser (Brilliant-Quantel, France), 5 ns pulse duration and $10 \mathrm{~Hz}$ repetition rate, capable of delivering about $850 \mathrm{~mJ}$ pulse energy at $1064 \mathrm{~nm}$ and $500 \mathrm{~mJ}$ at $532 \mathrm{~nm}$. The laser pulse energy was varied by varying the $Q$-switch delay through the AVASOFT software and was measured by an energy meter (Nova-Quantel, France). A quartz lens (convex) of $20 \mathrm{~cm}$ focal length was used to focus the laser beam on the target sample placed in air at atmospheric pressure. The measured diameter of the focused laser beam spot was about
$(0.10 \pm 0.01) \mathrm{cm}$; the focal spot area was $7.85 \times 10^{-3} \mathrm{~cm}^{2}$, which corresponds to a maximum laser fluence of $64 \mathrm{~J} \mathrm{~cm}^{-2}$. However, the emission spectra were recorded at varied fluence values from $1-50 \mathrm{~J} \mathrm{~cm}^{-2}$. To prevent the formation of deep craters, the sample was placed on a rotating stage for providing a fresh surface of the target for every laser shot. In order to prevent air breakdown in front of the sample, it was necessary to keep the distance between the lens and the sample less than the lens focal length. An optical fiber (high-OH, core diameter about $600 \mu \mathrm{m}$ ) was used to collect the plasma radiation with a collimating lens $\left(0-45^{\circ}\right.$ field of view), which was placed normal to the laser beam. The emitted radiation was captured by a set of four spectrometers (Avantes, Netherlands) each having $10 \mu \mathrm{m}$ slit width and covering the wavelength range of $250-870 \mathrm{~nm}$. To correct the emission signal, the dark signal was subtracted from the observed signal using the LIBS software. The same brass alloy was also quantitatively analysed by two other well-established techniques: EDX and LA-TOF-MS [33].

\section{Results and discussion}

The emission spectrum of the laser-produced brass plasma covering the spectral region $463-527 \mathrm{~nm}$ is presented in figure 1 . All the observed lines are identified as belonging to neutral copper and zinc. The three copper lines around 510-520 nm are due to the $3 \mathrm{~d}^{10} 4 \mathrm{p}{ }^{2} \mathrm{P}_{3 / 2} \rightarrow 3 \mathrm{~d}^{9} 4 \mathrm{~s}^{2}{ }^{2} \mathrm{D}_{5 / 2}$ at $510.55 \mathrm{~nm}, 4 \mathrm{~d}$ ${ }^{2} \mathrm{D}_{3 / 2} \rightarrow 4 \mathrm{p}{ }^{2} \mathrm{P}_{1 / 2}$ at $515.32 \mathrm{~nm}$ and $4 \mathrm{~d}{ }^{2} \mathrm{D}_{5 / 2} \rightarrow 4 \mathrm{p}{ }^{2} \mathrm{P}_{3 / 2}$ at $521.82 \mathrm{~nm}$ transitions. The triplet around $460-480 \mathrm{~nm}$ is identified as transitions from the $4 \mathrm{~s} 5 \mathrm{~s}{ }^{3} \mathrm{~S}_{1}$ upper level to the $4 \mathrm{~s} 5 \mathrm{~s}$ ${ }^{3} \mathrm{P}_{0,1,2}$ lower levels in zinc. Interestingly, the relative intensities of these zinc lines are in accordance with that expected in the LS coupling, proportional to the statistical weights of the terminating levels. The line at $465.11 \mathrm{~nm}$ is due to the transition from the $3 d^{9} 4 s 5 s{ }^{4} D_{7 / 2}$ upper level to the $3 d^{9} 4 s 5 p{ }^{4} F_{9 / 2}$ level in copper.

The first step in determining the elemental composition from the observed optical emission spectrum of the laser-produced brass plasma is to measure the relative line intensities and then construct a Boltzmann plot. We used the measured intensities and the relevant spectroscopic parameters of the 


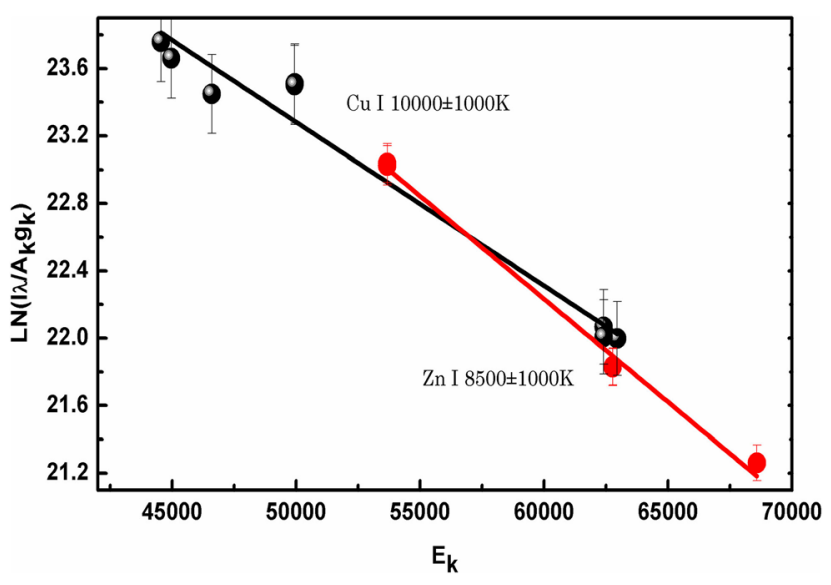

Figure 2. Typical Boltzmann plots for estimating the plasma temperatures from the $\mathrm{Cu} \mathrm{I}$ and $\mathrm{Zn}$ I spectral lines.

copper $(\mathrm{Cu} \mathrm{I})$ lines at $261.83 \mathrm{~nm}, 282.43 \mathrm{~nm}, 296.11 \mathrm{~nm}$, $319.40 \mathrm{~nm}, 427.51 \mathrm{~nm}, 458.69 \mathrm{~nm}, 465.11 \mathrm{~nm}, 510.55 \mathrm{~nm}$, $515.32 \mathrm{~nm}, 521.82 \mathrm{~nm}, 529.25 \mathrm{~nm}, 578.21 \mathrm{~nm}$ and that of $\mathrm{Zn} \mathrm{I}$ at $277.08 \mathrm{~nm}, 328.23 \mathrm{~nm}, 330.26 \mathrm{~nm}, 472.21 \mathrm{~nm}$ and $481.053 \mathrm{~nm}$ to draw the Boltzmann plot using the relation [34]:

$$
\ln \left(\frac{I \lambda}{h c A_{k} g_{k}}\right)=-\frac{E_{i}}{K_{\mathrm{B}} T}+\ln \left(\frac{N_{0}}{U(T)}\right)
$$

where $I$ is the intensity of the emission line, $\lambda$ is the transition wavelength, $h$ is Plank's constant, $c$ is the velocity of light, $A_{k}$ is the transition probability, $g_{k}$ is the statistical weight of the upper level, $E_{i}$ is the energy of the upper level, $K_{\mathrm{B}}$ is the Boltzmann constant, $T$ is the excitation temperature, $N_{0}$ is the total number density and $U(T)$ is the partition function. A plot of $\ln \left(\frac{I \lambda}{h c A_{k} g_{k}}\right)$ versus the upper level energies yields a straight line. The excitation temperature is calculated from its slope: $\frac{1}{K_{\mathrm{B}} T}$. The Boltzmann plots based on the $\mathrm{Cu} \mathrm{I}$ and $\mathrm{Zn} \mathrm{I}$ lines are presented in figure 2. The plasma temperatures were estimated as $(10000 \pm 1000) \mathrm{K}$ for $\mathrm{Cu} \mathrm{I}$ and $(8500 \pm 1000) \mathrm{K}$ for $\mathrm{Zn} \mathrm{I}$.

In order to determine the electron number density, we selected a well-isolated and Stark-broadened $\mathrm{Cu}$ I line at $465.11 \mathrm{~nm}$. The Stark width $\Delta \lambda_{\text {FWHM }}$ of this line was determined by de-convoluting the observed line profile as a Voigt profile, which takes into account the instrumental width, the Doppler width and the Stark broadening.

In figure 3, we present the experimental data points as dots and the line passing through the data points is the Voigt fit. The Stark width is related to electron number density as $[2,35]$ :

$$
N_{\mathrm{e}}=\left(\frac{\Delta \lambda_{\mathrm{FWHM}}}{2 \omega_{\mathrm{s}}}\right) \times 10^{6} .
$$

Here, $\Delta \lambda_{\text {FWHM }}$ is the full width at half maximum and $\omega_{\mathrm{s}}$ is the Stark-broadening parameter, and its value is reported in the literature as $4.1 \times 10^{-3} \mathrm{~nm}$ [35]. From the FWHM of the line profile, the electron number density is estimated as $(2.0 \pm 0.5) \times 10^{17} \mathrm{~cm}^{-3}$. The error in the electron number density is due to uncertainties in determining the FWHM and that in the Stark-broadening parameter.

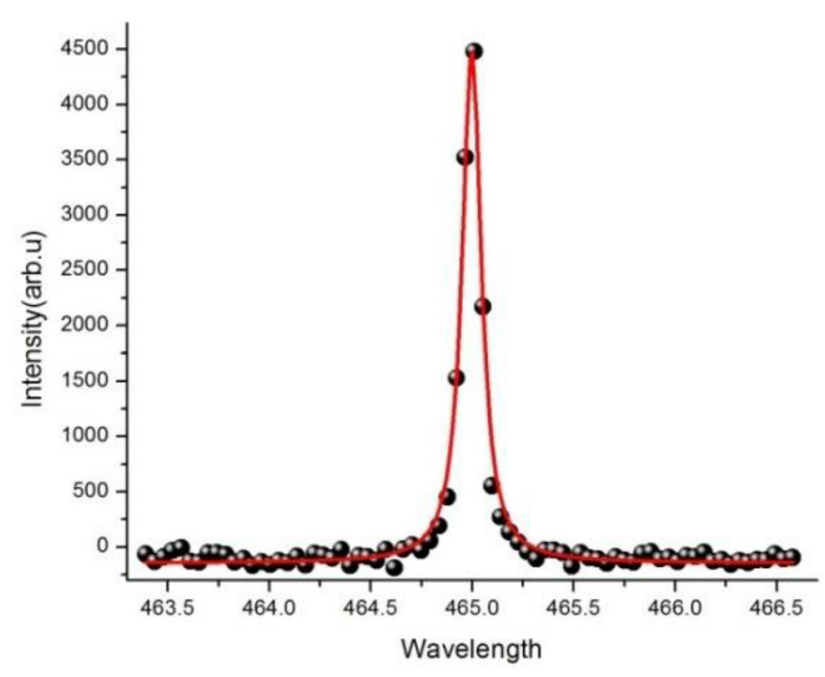

Figure 3. Stark-broadened line profile of the copper line at $465.01 \mathrm{~nm}$ along with the Voigt fit.

In order to use the LIBS technique for the quantitative analysis, it is mandatory that the laser-produced plasma is optically thin and holds the local thermodynamic equilibrium (LTE) condition. The validity of the optically thin plasma can be checked by comparing the observed line intensities of two lines of the same element and in the same charge state with that calculated from the known atomic parameters [36]:

$$
\frac{I_{1}}{I_{2}}=\frac{\lambda_{n m}}{\lambda_{k i}} \frac{A_{k i}}{A_{n m}} \frac{g_{k}}{g_{n}} \exp \left[-\frac{\left(E_{k}-E_{n}\right)}{k_{\mathrm{B}} T}\right]
$$

where $I_{1}$ and $I_{2}$ are the intensities of the lines at wavelengths $\lambda_{k i}, \lambda_{n m}$ with their corresponding transition probabilities from the upper levels to the lower levels $A_{k i}, A_{n m}$ respectively, $E_{k}$ and $g_{k}$ are the energy and statistical weight of the upper level corresponding to intensity $I_{1}$ and $E_{n}$, respectively, and $g_{n}$ is the energy and statistical weight of the upper level corresponding to intensity $I_{2}$. The left-hand side of the equation depends on the experimentally observed line intensities while the right-hand side contains atomic parameters that are tabulated in the NIST database [37]. In order to check the condition of the optically thin plasma, spectral lines having a common upper energy level or very close lying energy levels are selected to minimize the temperature dependence. Inserting the atomic parameters and the experimentally measured line intensities of $\mathrm{Zn} \mathrm{I}$ at $636.23 \mathrm{~nm}$ and $334.50 \mathrm{~nm}$ in equation (3) yields the values 0.538 and 0.582 , respectively, which are in good agreement, and differ by only $7 \%$. Such calculations for other pairs of lines, $\mathrm{Cu}$ I at $(427.51 \mathrm{~nm}, 465.11 \mathrm{~nm}), \mathrm{Cu}$ II at $(268.93 \mathrm{~nm}, 271.35 \mathrm{~nm})$ and $\mathrm{Zn}$ II $(250.19 \mathrm{~nm}, 255.79 \mathrm{~nm})$, also agree within the experimental errors. The difference in the calculated values is associated to the errors in measuring the line intensities and that in the reported transition probabilities. In addition, the triplet due to transitions from the $4 \mathrm{~s} 5 \mathrm{~s}^{3} \mathrm{~S}_{1}$ upper level to the $4 \mathrm{~s} 5 \mathrm{~s}{ }^{3} \mathrm{P}_{0,1,2}$ lower levels in zinc also possesses intensities according to their statistical weights. Thus, this supports our assertion that the plasma can be considered as optically thin. 
The condition of the local thermodynamic equilibrium was validated using the Mc-Whiter ccriteria [38]. The lower limit of the electron number density under this condition can be determined from the relation:

$$
N_{\mathrm{e}} \geqslant 1.6 \times 10^{12} T^{1 / 2}(\Delta E)^{3}
$$

where $T(\mathrm{~K})$ is the plasma temperature and $\Delta E(\mathrm{eV})$ is the energy difference between the upper and the lower level. The plasma having an electron number density greater than that deduced from the above relation can be considered as close to fulfilling the LTE condition. The lower limit of the electron number density at an elevated temperature and energy difference between the transitions is calculated using equation (4) as $2.0 \times 10^{13} \mathrm{~cm}^{-3}$, which is much lower than that $\left((2.0 \pm 0.5) \times 10^{17} \mathrm{~cm}^{-3}\right)$ calculated from the Starkbroadened line profile of the copper line at $29.26 \mathrm{~nm}$. Thus the plasma in the present studies can be considered very close to LTE.

\subsection{Quantitative analysis by CF-LIBS: Boltzmann plot method}

As we have established that the plasma is optically thin and also fulfils the condition of LTE (see above), we therefore used the data for the qualitative analysis based on the CF-LIBS and the Boltzmann plot method. The intensity $\overline{I_{b a}}$ of a spectral line is related to the transition between two levels $E_{b}$ and $E_{a}$ and can be estimated from the relation:

$$
\overline{I_{b a}}=F C_{\mathrm{s}} A_{b a} \frac{g_{b}}{U(T)} \mathrm{e}^{\frac{-E_{b}}{k_{\mathrm{B}} T}}
$$

where $A_{b a}$ is the transition probability connecting the upper level $E_{b}$ and the lower level $E_{a}, g_{b}$ is the statistical weight of the upper level, $F$ is the experimental factor, $k_{\mathrm{B}}$ is the Boltzmann constant, $U(T)$ is the partition function at temperature $T$ and $C_{\mathrm{s}}$ is the concentration of the atomic species. Taking the logarithm on both sides of equation (5):

$$
\ln \left[\frac{\overline{I_{b a}}}{A_{b a} g_{b}}\right]=-\frac{E_{b}}{k_{\beta} T}+\ln \left[\frac{F C_{\mathrm{s}}}{U(T)}\right] .
$$

This equation can be written as a linear equation:

$$
Y=m x+q_{\mathrm{s}}:
$$

$Y=\ln \left[\frac{\overline{I_{b a}}}{A_{b a} g_{b}}\right], x=E_{b}, m=-\frac{1}{T k_{\mathrm{B}}}$ and $q_{\mathrm{s}}=\ln \left[\frac{F C_{\mathrm{s}}}{U(T)}\right]$

where $q_{\mathrm{s}}$ is the intercept along the $Y$-axis of the Boltzmann Plot and its slop, $\mathrm{m}$, is related to the electron temperature. If the laser-produced plasma is close to LTE condition, then the slops of the Boltzmann plots for each element should be approximately the same and the intercepts may differ according to the concentration of that element in the sample. Simplifying equation (6), we can determine the experimental factor and the elemental concentration using the following relation:

$$
F C_{\mathrm{s}}=U(T) \mathrm{e}^{q_{\mathrm{s}}}
$$

where $F$ is the experimental factor, which can be obtained by normalizing the sum of the concentration of all the species to unity, and the concentration of the neutral species can be extracted from this relation. Boltzmann plots for $\mathrm{Cu} \mathrm{I}$ and $\mathrm{Zn}$ I are presented in figure 2. The concentrations of the neutral species were calculated from the intercepts along the $Y$-axis.

To calculate the concentration of the ionized species, the Saha-Boltzmann equation was used,which relates the number densities of the neutral as well as the singly ionized species [17]:

$$
\frac{n_{z+1}^{\alpha}}{n_{z}^{\alpha}} N_{\mathrm{e}}=6.04 \times 10^{21} T_{e v}^{3 / 2} \frac{U_{z+1}^{\alpha}}{U_{z}^{\alpha}} \exp \left(-\frac{\chi_{z}^{\alpha}}{T_{e v}}\right) \mathrm{cm}^{-3}(10)
$$

where $N_{\mathrm{e}}$ is the electron number density in the plasma, $n_{z+1}^{\alpha}$ and $n_{z}^{\alpha}$ are the number densities of the neutral and the singly ionized species respectively, $T(\mathrm{eV})$ is the plasma temperature, $U_{z+1}^{\alpha}$ and $U_{z}^{\alpha}$ are the partition functions, and $\chi_{z}^{\alpha}$ is the ionization energy of the species. From this equation, we determined the number density of the ionized species, whereas the number density of the neutral species was calculated from equation (9) (see above); thus the total concentration is represented as sum of the neutral and ionized concentrations of each element [10]:

$$
C_{\mathrm{T}}=C_{\mathrm{s}}^{\mathrm{I}}+C_{\mathrm{s}}^{\mathrm{II}} .
$$

This procedure yields the concentration of $\mathrm{Cu}$ as $70 \%$ and that of $\mathrm{Zn}$ as $30 \%$ containing about $20 \%$ error.

\subsection{Quantitative analysis by the CF-IRSAC method}

To correct the observed intensities of the spectral lines Sun et al [22] proposed a relation to calculate the self-absorption coefficient using the following relation

$$
I^{b a}=f_{\lambda}^{b} F C_{\mathrm{S}} A_{b a} \frac{g_{b}}{U(T)} \mathrm{e}^{-\frac{E_{b}}{k_{\mathrm{B}} T}}
$$

where $f_{\lambda}^{b}$ is the self-absorption coefficient that varies from zero to one. If the self-absorption coefficient $f_{\lambda}^{b}=0$, this reflects that the spectral line is completely reabsorbed, and if $f_{\lambda}^{b}=1$, this means that the spectral line is unaffected by selfabsorption. The spectral lines having low excitation energies in the upper level and higher transition probability are strongly affected by self-absorption. On the other hand, self-absorption is low for those lines that have high excitation energy in the upper level and low transition probability. The $\mathrm{Cu}$ I line at $529.25 \mathrm{~nm}$ and $\mathrm{Zn}$ I line at $328.23 \mathrm{~nm}$ were selected as internal reference lines and the self-absorption coefficient for the other spectral lines were calculated using the following relation [22].

$$
\frac{f_{\lambda}^{b}}{f_{\lambda R}^{b}}=\frac{I_{\lambda}^{b a} A_{m n} g_{m}}{I_{\lambda R}^{m n} A_{b a} g_{b}} \mathrm{e}^{-\frac{\left(E_{m}-E_{b}\right)}{k_{\mathrm{B}} T}}
$$

where $A_{m n}$ is the transition probability, $g_{m}$ is the statistical weight of the upper level, $I_{\lambda R}^{m n}$ and $E_{m}$ are the line intensity and the energy of the selected internal reference line, and $A_{b a}, g_{b}, I_{\lambda}^{b a}$ and $E_{b}$ are the transition probability, statistical weight and intensity of the other lines in the spectrum. 
We selected an internal reference line having high excitation energy in the upper level $E_{m}$ and low transition probability $A_{m n}$ for which the self-absorption coefficient is taken as one, $f_{\lambda}^{b} \approx 1$. The self-absorption coefficient depends on the laser ablated plasma temperature, which is initially deduced from the Boltzmann plot method. The selection of an internal reference line is a key point of the IRSAC method because the internal reference line plays an important role in extracting the self-absorption coefficient. It is possible that the self-absorption coefficient may turn out to be greater than one. There are many reasons behind this situation such as selection of the wrong internal reference line or the presence of self-absorption in the reference line [21, 22].

The self-absorption coefficients for all the other lines can be calculated with the help of the internal reference line using the relation:

$$
f_{\lambda}^{b}=\frac{I_{\lambda}^{b a} A_{m n} g_{m}}{I_{\lambda R}^{m n} A_{b a} g_{b}} \mathrm{e}^{-\frac{\left(E_{m}-E_{b}\right)}{k_{\mathrm{B}} T}} .
$$

To get the corrected line intensities, the self-absorption coefficient was divided by the observed line intensities using the relation:

$$
\widehat{I}_{\lambda}^{b a}=\frac{I_{\lambda}^{b a}}{f_{\lambda}^{b}}=\frac{I_{\lambda R}^{m n} A_{b a} g_{b}}{A_{m n} g_{m}} \mathrm{e}^{-\frac{\left(E_{m}-E_{b}\right)}{k_{\mathrm{B}} T}} .
$$

This is an iterative procedure suggested by Sun et al [22] that has been adopted here.

\subsection{Algorithm of the IRSAC method}

The IRSAC method is based on an iterative procedure, following equations (14) and (15), which needs to be repeated until the correct line intensities are achieved. The following steps are followed in the IRSAC procedure.

- Step 1. Select an internal reference line for each element.

- Step 2. Calculate appropriate values of the reference lines, which depend on the Boltzmann plot.

- Step 3. With the help of the Boltzmann plot, calculate the plasma temperature.

- Step 4. Calculate the self-absorption coefficient, $f_{\lambda}^{b}$.

- Step 5. Find out the corrected line intensities.

- Step 6. If the equating coefficient converges then proceed further, otherwise again calculate the temperature and repeat this procedure until it converges.

- Step 7. After convergence, check whether the temperature difference between all species is lower than $10 \%$.

- Step 8. If the difference is lower than $10 \%$ then calculate their elemental compositions using the CF-LIBS procedure.

- Step 9. If the difference is greater than $10 \%$ then set the highest measured temperature as a mean value of the plasma temperature.

- Step 10. Again calculate the self-absorption coefficient and repeat the steps until the temperature difference between all the elements is less than $10 \%$.

The self-absorption coefficient was calculated using the lines of $\mathrm{Cu}$ I at $529.25 \mathrm{~nm}$ and $\mathrm{Zn}$ I at $328.23 \mathrm{~nm}$. The intensities

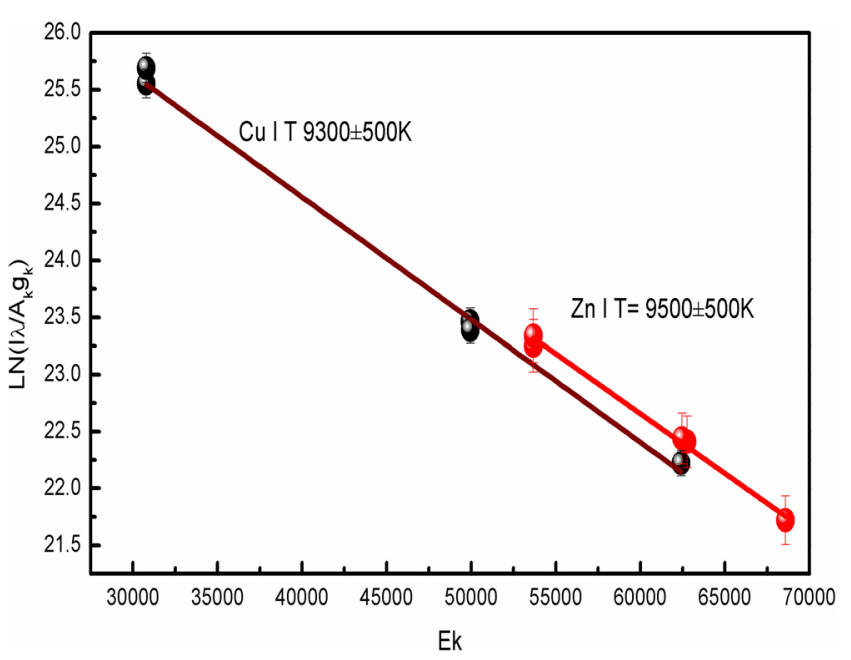

Figure 4. Typical Boltzmann plots of copper and zinc after selfabsorption corrections to calculate the plasma temperatures.

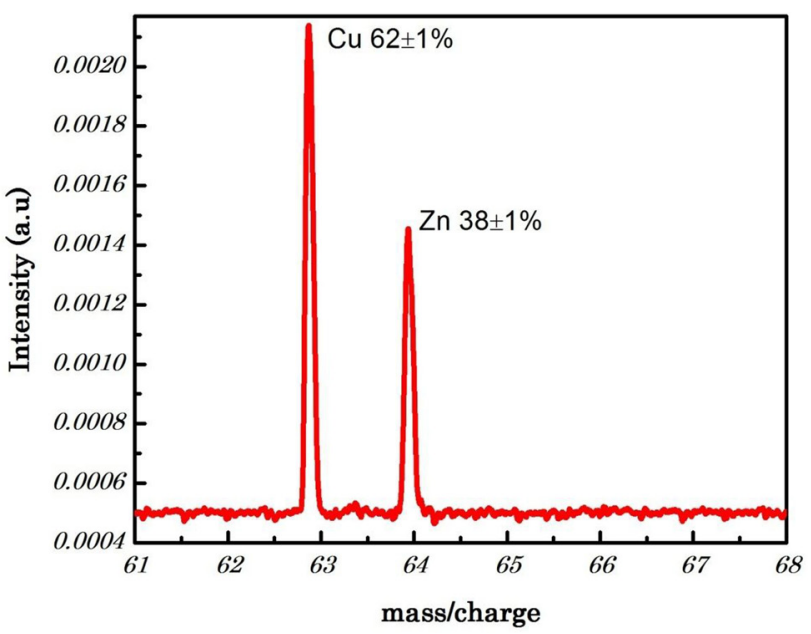

Figure 5. The mass spectrum of a brass alloy measured by LATOF-MS

of the other lines were corrected following the above-mentioned procedure. The corrected line intensities were used to redraw the Boltzmann plots, as shown in figure 4. Some $\mathrm{Cu}$ I lines were not affected by self-absorption, but the correction process softly changed their intensities. On the other hand, the lines at $261.82 \mathrm{~nm}$ and $282.42 \mathrm{~nm}$ were corrected by this process. Consequently, the data points were now more regular and followed the fitted lines in the Boltzmann plot.

After the intensity corrections using the IRSAC method, the plasma temperatures calculated by the Boltzmann plot (see figure 4) using the $\mathrm{Cu}$ I and $\mathrm{Zn}$ I lines approached comparable values. From the intercepts, this method yields the concentration of $\mathrm{Cu}$ as $63.4 \%$ and that of $\mathrm{Zn}$ as $36.6 \%$ with about $3 \%$ error. The results of the compositional analysis are more precise as compared to that of the basic CF-LIBS.

\subsection{Quantitative analysis by LA-TOF-MS}

A compositional analysis of the $\mathrm{Cu}-\mathrm{Zn}$ alloy was also performed using the LA-TOF-MS and EDX techniques. The spectrum acquired with the TOF-MS, a one-metre linear system $[5,33]$ is shown in figure 5. From the observed ion signals, 
Table 1. Quantitative results for the copper-zinc based brass alloy.

\begin{tabular}{|c|c|c|c|c|c|c|}
\hline \multirow[b]{2}{*}{ Element (certified) } & \multirow[b]{2}{*}{ LA-TOF $(\%)$} & \multirow[b]{2}{*}{$\operatorname{EDX}(\%)$} & \multicolumn{2}{|c|}{ Weight $(\%)$ concentration } & \multicolumn{2}{|c|}{ Relative standard error (\%) } \\
\hline & & & CF-LIBS (\%) & IRSAC $(\%)$ & CF-LIBS (\%) & $\operatorname{IRSAC}(\%)$ \\
\hline Copper $(62 \%)$ & 62 & 61.75 & 70 & 63.36 & 12 & 2 \\
\hline Zinc $(38 \%)$ & 38 & 38.25 & 30 & 36.64 & 21 & 4 \\
\hline
\end{tabular}

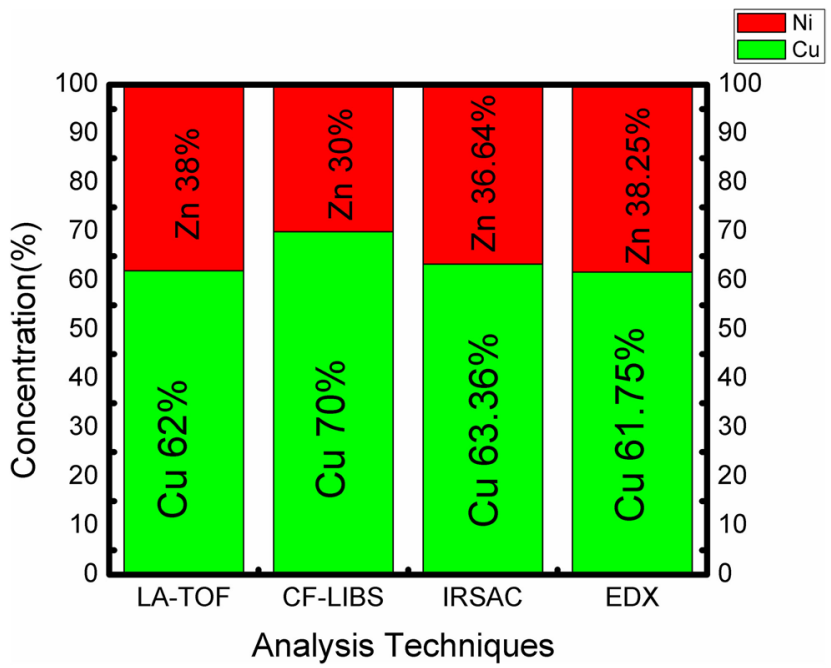

Figure 6. A histogram of the results of the composition of the copper-zinc based brass alloy acquired using different analytical techniques.

the elemental composition was determined from the integrated line intensity as: $\mathrm{Cu}(62 \%)$ and $\mathrm{Zn}(38 \%)$. These values are in excellent agreement with that of the certified compositions.

The elemental analysis was also achieved by EDX. The analysis yielded the major elemental composition $\mathrm{Cu}$ $(61.75 \%)$ and $\mathrm{Zn}(38.25 \%)$.

A comparison of the elemental compositions of the brass alloy, determined by the LIBS based techniques, LA-TOFMS and EDX is presented in table 1. In CF-LIBS, using the basic Boltzmann plot method without self-absorption correction, the estimated error is greater than $10 \%$. The IRSAC yielded results of compositional analysis comparable with that of LA-TOF-MS and EDX (error within 2\%) as well as with the actual composition.

In figure 6 we show a comparison of the quantitative analysis results of LS-TOF-MS and CF-LIBS using basic Boltzmann plot method, the internal reference line self-absorption correction method, and EDX. It is evident from the histogram that the IRSAC technique makes the CF-LIBS technique more reliable after considering the self-absorption effects.

\section{Conclusion}

In this study, we presented the elemental composition of a copper-zinc based brass alloy of certified composition $(62 \% \mathrm{Cu}$ and $38 \% \mathrm{Zn}$ ) using the LIBS techniques as well as with other established analytical techniques. The two LIBS techniques were based on the Boltzmann plot method (CF-LIBS) and an internal reference line self-absorption correction method (IRSAC). The error estimated in the basic Boltzmann plot method was around
$10 \%$, whereas the IRSAC method took into account the selfabsorption effect, which reduced the errors to about $5 \%$. The compositions extracted using LA-TOF-MS and EDX contained much smaller errors (about 1\%), which were also in excellent agreement with that of the certified composition. It was concluded that the LIBS based IRSAC method is a reliable method for the quantitative analysis of any type of material.

\section{Acknowledgments}

We are grateful to the Pakistan Academy of Sciences for the financial assistance to acquire the Laser system and the Higher Education Commission of Pakistan for the indigenous PhD scholarship to Mr Nasar Ahmed.

\section{References}

[1] Cremers D A and Radziemski D A 2006 Handbook of Laser Induced Breakdown Spectroscopy (New York: Wiley)

[2] Noll R, Begemann C F, Brunk M, Connemann S, Meinhardt C, Scharun M, Sturm V, Makowe J and Gehlen C 2014 Spectrochim. Acta B 93 41-51

[3] Bassiotis I, Diamantopoulou A, Giannoudakos A, RoubaniKalantzopoulou F and Kompitsa M 2001 Spectrochim. Acta B 56 671-83

[4] Shaikh N M, Rashid B, Hafeez S, Jamil Y and Baig M A 2006 J. Phys. D: Appl. Phys. 391384

[5] Ahmed N, Ahmed R, Rafiqe M and Baig M A 2017 Laser Part. Beams 35 1-9

[6] Noll R, Bette H, Brysch A, Kraushaar M, Monch I, Peter L and Sturm V 2001 Spectrochim. Acta B 56 637-49

[7] Cristoforetti G, Legnaioli S, Palleschi V, Salvetti A, Tognoni E, Benedetti P A, Brioschiand F, Ferrario F and Anal J 2006 At. Spectrom. 21 697-702

[8] Rashid B, Ahmed R, Ali R and Baig M A 2011 Phys. Plasmas 18 073301-7

[9] Tognoni E, Cristoforetti G, Legnaioli S, Palleschi V, Salvetti A, Mueller M, Panne U and Gornushkin I 2007 Spectrochim. Acta B 62 1287-302

[10] Fichet P, Menut D, Brennetot R, Vors E and Rivoallan A 2003 Appl. Optics 42 6029-35

[11] Gupta G P, Suri B M, Verma A, Sunderaraman M, Unnikrishnan V K, Alti K, Kartha V B and Santhosh C 2011 J. Alloys Compd $\mathbf{5 0 9} 3740-5$

[12] Gaudiuso R, Dell'Aglio M, De Pascale O, Loperfido S, Mangone A and De'Giacomo A 2014 Anal. Chim. Acta 813 15-24

[13] Kalmhofer P J, Eschlbock-Fuchs S, Huber N, Rossler R, Heitz J and Pedaening J D 2015 Spectrochim. Acta B 106 67-74

[14] Zhanga S, Wanga X, Hea M, Jianga Y, Zhanga B, Hanga W and Huanga B 2014 Spectrochim. Acta B 97 13-33

[15] Miziolek A W, Pallesschi V and Schecchter I 2006 Laser-Induced Breakdown Spectroscopy: Fundamentals and Application (Cambridge: Cambridge University Press) 
[16] Ciucci A, Corsi M, Palleschi V, Rastelli S, Salvetti A and Tognoni E 1999 Appl. Spectrosc. 53 960-4

[17] Gomba J M, Angelo C D and Bertuccelli D 2001 Spectrochim. Acta B 56 695-705

[18] Abbass Q, Ahmed N, Ahmed R and Baig M A 2016 Plasma Chem. Plasma Process. 36 1287-99

[19] Winefordner J D, Gornushkin I B, Correll T, Smith B W and Omenetto N 2004 J. Anal. At. Spectrom. 1910611083

[20] Noll R 2012 Laser-Induced Breakdown Spectroscopy —Fundamentals and Applications (Berlin: Springer)

[21] Dong J, Liang L, Wei J, Tang H, Zhang T, Yang X, Wang K and Li H 2015 J. Anal. At. Spectrom. 30 1336-44

[22] Sun L and Yu H 2009 Talanta 79 388-95

[23] El Sherbini A M, Hegazy H, Cristoforetti G, Legnaioli S, Palleschi V, Pardini L, Salvetti A and Tognoni E 2005 Spectrochim. Acta B 60 1573-9

[24] Achouri M, Hamed T B, Beldjilali S A and Belasri A 2015 Plasma Phys. Rep. 41 758-68

[25] Andrade J M, Cristoforetti G, Legnaioli S, Lorenzetti G, Palleschi V and Shaltout A A 2010 Spectrochim. Acta B $\mathbf{6 5} 658-63$

[26] Shaikh N M, Hafeez S, Kalyar M A, Ali R and Baig M A 2008 J. Appl. Phys. 104103108
[27] Grifoni E, Legnaioli S, Lorenzetti G, Pagnotta S, Poggialini F and Palleschi V 2016 Spectrochim. Acta B 124 40-6

[28] Shaltout A A, Mostafa N Y, Abdel-Aal M S and Shaban H A 2010 Eur. Phys. J. Appl. Phys. 5011003

[29] Ahmed N, Umar Z A, Ahmed R and Baig M A 2017 Spectrochim. Acta B 136 39-44

[30] Baig M A, Qamar A, Fareed M A, Anwar-ul-Haq M and Ali R 2012 Phys. Plasma 19063304

[31] Ahmed R, Ahmed N, Iqbal J and Baig M A 2016 Phys. Plasmas 23083101

[32] Iqbal J, Ahmed R and Baig M A 2017 Laser Phys. 27046101

[33] Ahmed N, Ahmed R, Umar Z A and Baig M A 2017 Laser Phys. 27086001

[34] Borgia I, Burgio L M F, Corsi M, Fantoni R, Palleschi V, Salvetti A, Squarcialupi M S and Tognoni E $2000 \mathrm{~J}$. Cult. Herritage 1 281-6

[35] Konjevic N 1990 J. Phys. Chem. Ref. Data 313

[36] Unnikrishnan V K, Mridul K, Nayak R, Alti K, Kartha V B, Santhosh C and Gupta G P 2012 Pramana-J. Phys. 79 299-310

[37] NIST Atomic Spectra Database (http://physics.nist.gov)

[38] Cristoforetti G, DeGiacomo A, Dell'Aglio M, Legnaioli S, Tognoni E, Palleschi V and Omenetto N 2010 Spectrochim. Acta B 65 86-95 